\title{
Image Fusion Method Based on Edge Feature Detection in Electrical Capacitance Tomography
}

\author{
Chen Deyun, Gao Ming, Song Lei, Lin Jianan, Yao Yumei and Wei Li \\ Higher Educational Key Laboratory for Measuring and Control Technology and \\ Instrumentations of Heilongjiang Province, Harbin University of Science and \\ Technology, Harbin 150080, China \\ E-mail: chendeyun@hrbust.edu.cn
}

\begin{abstract}
"Soft-field" nature and ill-posed problems to influence on the accuracy of image reconstruction in electrical capacitance tomography technology (ECT), in the analysis of ECT basic principle and imaging algorithm, a novel image fusion method based on edge feature detection using the feature of different frequency domains of wavelet decomposition is presented in this paper. The LBP algorithm and Landweber algorithm are used as the base of image reconstruction in the paper, the fusion rules based on the combination of Local gradient and local signal strength and the weighted averaging operator are used to fuse the high-frequency coefficients and low-frequency coefficients of the fused image, in this way, higher accuracy imaging results can be got. The simulation results show that the image fusion accuracy is improved, and reconstruction image effect is enhanced.
\end{abstract}

Keywords: Electrical Capacitance Tomography; image reconstruction; image fusion; local gradient; signal strength

\section{Introduction}

Electrical capacitance tomography (ECT) is a new type of computer tomography technology which is formed and developed in the 1980s, it always have many advantages such as non-invasive, simple structure, low cost, fast response, good safety performance ,applicable to a wide range and so on; we also can extract flow type and phase holdup from it's measurement signal and it is a hot research topic in the Process Tomography (PT)[1].The detection effects of ECT depends largely on the access of the reconstructed image and ECT solution is less stable and has a seriously sick due to the non-linear and "soft field" nature. The LBP algorithm, the regular method, Landweber iteration method and the CG algorithm are used ECT reconstruction algorithm; these algorithms have their own imaging characteristics, but at the same time have their own limitations [2].

Image fusion is a data fusion technology whose main object of study is image, it always can be saw as a process that lets the multiple images of the same target which is obtained from one or more sensors to synthesis an image used the image fusion algorithm. A more comprehensive and reliable description can be obtained on the same imaging objects from the way to extract image information from multiple source in order to achieve further analysis and understanding of the characteristics of the image and a new way of thinking to solve some problems of electrical capacitance tomography can be provided by the above method. 
An image fusion method based on edge characteristics in electrical capacitance tomography is put forward in this paper and the LBP algorithms, Landweber iterative method are used as the reconstructed image's source.

\section{Principle of the Electrical Capacitance Tomography System}

As shown in Figure 1, the ECT system consists of capacitance sensor, data acquisition system and imaging computer [3]. By contrasting the measuring voltage of actual object's boundary with the measuring voltage of the corresponding location at the state that pipe is full of water and using appropriate imaging algorithm, we can achieve the result of the visual measurement.

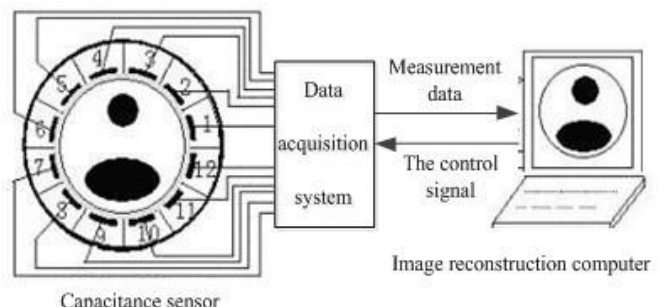

Figure 1. System Components of Capacitance Tomography

The image reconstruction problems of ECT is based on the linear model that the mapping of dielectric constant and capacitance. The discrete, linearly, normalized model is shown in the following formula [4]:

$$
C=S G
$$

In this formula, $C \in R^{m}$ is the normalized capacitive vector, $S \in R^{m \times n}$ is the coefficient matrix (sensitivity matrix), $G \in R^{n}$ and is the normalized distribution image vector of media.

The technology of ECT image reconstruction is to obtain measurement values from the plurality of electrodes then to represent itself by the different grayscale values. The common practice is to let the large dielectrics to correspond to gray value items then reconstruct a cross-sectional view of the multiphase flow with these gray values.

In support of this principle, Landweber iterative method, conjugate gradient (CG) method and other image reconstruction algorithms have been proposed.

\section{Principle of Algorithm}

\subsection{Wavelet Multi-resolution Analysis}

The wavelet transform is used as the multi-resolution analytical tool, using the wavelet transform in this paper, signal can be decomposed into low-frequency approximate component (scale coefficient) and high-frequency detail component (wavelet coefficient).

Using the rapid wavelet transform algorithm which is proposed by Mallat, images (twodimensional signal) can be decomposed at the $2^{j}$ resolution [5]:

$$
C_{j+1}=C_{j} H_{r} H_{c}, D_{j+1}^{1}=C_{j} H_{r} G_{c}, D_{j+1}^{2}=C_{j} G_{r} H_{c}, D_{j+1}^{3}=C_{j} G_{r} G_{c}
$$

Indicate the horizontal direction detail component, vertical direction detail component and diagonal direction detail component at this resolution, $H_{r} 、 H_{c}$ respectively indicate the row direction low pass filter and column direction low-pass filter, $G_{r} 、 G_{c}$ respectively indicate the 
row direction low pass filter and column direction high-pass filter, The two-dimensional reconstruction of wavelet is decomposition of the inverse process:

$$
C_{j}=C_{j+1} H_{r}^{*} H_{c}^{*}+D_{j+1}^{1} H_{r}^{*} G_{c}^{*}+D_{j+1}^{2} G_{r}^{*} H_{c}^{*}+D_{j+1}^{3} G_{r}^{*} G_{c}^{*}
$$

$H^{*} 、 G^{*}$ Respectively indicate the conjugate transpose matrix of $H 、 G$.

Based on the above wavelet decomposition principle [6], the fusion algorithm put forward by this paper is completed in the following steps:

1. Using formula (2), the reconstructed images of different sources can be decomposed into scaling coefficients and the wavelet coefficient of three directions.

2. Using different fusion rules for the fusion process of fused image's wavelet coefficient and scale coefficient.

3. Using formula (3) for the inverse wavelet transform, the fused image can be obtained after reconstruction.

\subsection{The Selection of Characteristic Parameters}

The edges of the image has its local characteristics[7], so local image parameters can better reflect the image edge features, this article uses the local gradient and local signal strength to reflect the image edge information.

(1) Local gradient. The change of the mount's direction and speed is reflected by the gradient. The local gradient of the image represents the change of the gray value in one direction of the image. The local gradient is defined as the following formula:

$$
G=\frac{1}{M \times N} \sum_{i=1}^{M} \sum_{j=1}^{N} \sqrt{\Delta x f(i, j)^{2}+\Delta y f(i, j)^{2}}
$$

In this formula, $\Delta x f(i, j)$ and $\Delta y f(i, j)$ respectively indicate the gradient at point $(i, j)$ on the $x$ and $y$ directions, $M \times N$ indicates the size of the region of the window. Be seen, if the local edge is present, the larger value of the gradient can be calculated when the local gray value sharply changes.

(2) Local signal strength. Local signal strength describes the number of useful information provided by the detail component $D$ in the corresponding window region; it can be defined as the following formula:

$$
I_{j, S}^{\varepsilon}(m, n)=\sum_{m^{\prime} \in M, n^{\prime} \in N} P\left(m^{\prime}, n^{\prime}\right)\left|D_{j, S}^{\varepsilon}\left(m+m^{\prime}, n+n^{\prime}\right)\right|
$$

In this formula, $D_{j, S}^{\varepsilon}(1 \leq \varepsilon \leq 3)$ indicates the wavelet coefficient at the $2^{j}$ resolution, $P$ is a window whose size is $M \times N$. The size of window used in the experiment is $3 \times 3$. The local signal strength much greater the useful details that the region can provide much more.

\subsection{The Fusion Rules of All the Frequencies}

Rational design of data fusion strategy is the key to get high-quality fused image, using different fusion rules in different frequencies domain to obtain a multi-resolution analysis [8] of the synthesized image and thereby to retain the significant feature of the original image in the synthesized image at different frequencies. Combined with the ECT imaging characteristics, in the following, the design of different image fusion rules for the highfrequency domain and low-frequency domain will be introduced.

\subsubsection{High-frequency Component of the Fusion Rules}


The image wavelet coefficients (high frequency) can reflect the catastrophe characteristics of the image, that the edge of the source image and area boundary feature, combined with the imaging characteristics of ECT, the design that the part of image wavelet coefficient's fusion rule is clearly more critical.

Combining with the gradient and the signal strength, not only the overall characteristics of the local window can be reflected but also the independent information of each pixel within the window can be considered. So the edge feature degree of fusion parameter GI is designed as the following formula:

$$
G I=G \times I
$$

In the above formula, $G$ indicates local gradient, $I$ indicates the local signal strength. Through the local gradient multiplying the local signal strength, not only the reflection of the local gradient and the local signal strength to the local information and edge features in the image can be enhanced but also the shortcomings that the local gradient is easily impacted by independent noise and the shortcoming that the local signal strength can't accurate enough to reflect the edge information can be compensated.

When the wavelet coefficient (high frequency component) is fused, a local window is used as a object being investigated to calculate the statistical characteristics of the local area. Because significant correlation exists between the pixels of the image, so examine the image within a region to a single pixel ratio investigated object to better reflect the characteristics and trend of the image. The window's size which is adopted in this paper is $3 \times 3$, on the basis of this paper, designs the fusion rule of high-frequency part based on the image edge feature is designed as follows:

In $D_{j+1}^{L}$ and $D_{j+1}^{L}$ that the high frequency components of image $\mathrm{L}$ and image $\mathrm{H}$, the edge significant degree that $D_{j+1}^{L}$ and $D_{j+1}^{H}$ for each pixel within the window region are calculated, and then the process that the normalization of $D_{j+1}^{L}$ and $D_{j+1}^{H}$ is introduced as the following formula:

$$
\begin{aligned}
& G I_{D}^{L}(m, n)=\frac{G I^{L}(m, n)}{G I^{L}(m, n)+G I^{H}(m, n)} \\
& G I_{D}^{H}(m, n)=\frac{G I^{H}(m, n)}{G I^{L}(m, n)+G I^{H}(m, n)}
\end{aligned}
$$

In the above formula, $G I_{D}^{H}(m, n)$ and $G I_{D}^{L}(m, n)$ indicate the normalized edge feature degree of each point's High-frequency components in image $\mathrm{L}$ and image $\mathrm{H}$.

Then, for each point in the $D_{j+1}^{L}$ and $D_{j+1}^{H}$, if $\left|G I_{D}^{H}(m, n)-G I_{D}^{L}(m, n)\right|>T$, images will be fused in accordance with the maximum rule:

$$
D_{j+1}^{F}(m, n)=\left\{\begin{array}{l}
D_{j+1}^{L}, G I_{D}^{L}(m, n)>G I_{D}^{H}(m, n) \\
D_{j+1}^{H}, G I_{D}^{H}(m, n)>G I_{D}^{L}(m, n)
\end{array}\right.
$$

If $\left|G I_{D}^{H}(m, n)-G I_{D}^{L}(m, n)\right|<T$ images will be fused in accordance with the weighted average rule:

$$
D_{j+1}^{F}(m, n)=\frac{D_{j+1}^{L}(m, n)+D_{j+1}^{H}(m, n)}{2}
$$


Wherein $T$ is a threshold value, in this paper $T$ is used as 0.3 , through the establishment of the threshold value we can more fully utilize the information of the source image, to avoid fusion of the image information from a source image completely.

\subsubsection{Low-frequency Component of The Fusion Rules}

In the outline of the image is reflected by the scale coefficient (low frequency component), the correct choice of scaling coefficient has an important role to improve the visual effect of the image.

In $I^{L}$ and $I^{H}$ that the high frequency components of image $\mathrm{L}$ and image $\mathrm{H}$, the edge significant degrees that $G I^{L}$ and $G I^{H}$ for each pixel within the window region are calculated in this paper, and then the process that the normalization of $G I^{L}$ and $G I^{H}$ is introduced as the following formula:

$$
\begin{aligned}
\overline{G I_{j+1}^{L}} & =\frac{\sum_{m} \sum_{n} G I^{L}(m, n)}{\sum_{m} \sum_{n} G I^{L}(m, n)+\sum_{m} \sum_{n} G I^{H}(m, n)} \\
\overline{G I_{j+1}^{H}} & =\frac{\sum_{m} \sum_{n} G I^{H}(m, n)}{\sum_{m} \sum_{n} G I^{L}(m, n)+\sum_{m} \sum_{n} G I^{H}(m, n)}
\end{aligned}
$$

In the above formula, $\overline{G I_{j+1}^{L}}$ and $\overline{G I_{j+1}^{H}}$ indicate the ratio of scale factor edge features of the image $\mathrm{L}$ and image $\mathrm{H}$. Then, using the normalized edge feature, the data fusion rules used as the following:

$$
I^{F}=\overline{G I_{j+1}^{L}} I^{L}+\overline{G I_{j+1}^{H}} I^{H}
$$

The data fusion used this rule can take full advantage of the complementary information in different images, so as to get the best results of the fused image.

LBP algorithm and Landweber iterative method was chosen as the ECT image reconstruction algorithm and the images obtained from the rebuilding of these algorithms are used as the source image for image fusion. The Wave Toolbox in MATLAB is selected to operate the experiment, the decomposition level is set to 5 , the threshold $\mathrm{T}=0.3$. The experimental procedure is shown in the following Figure 2: 


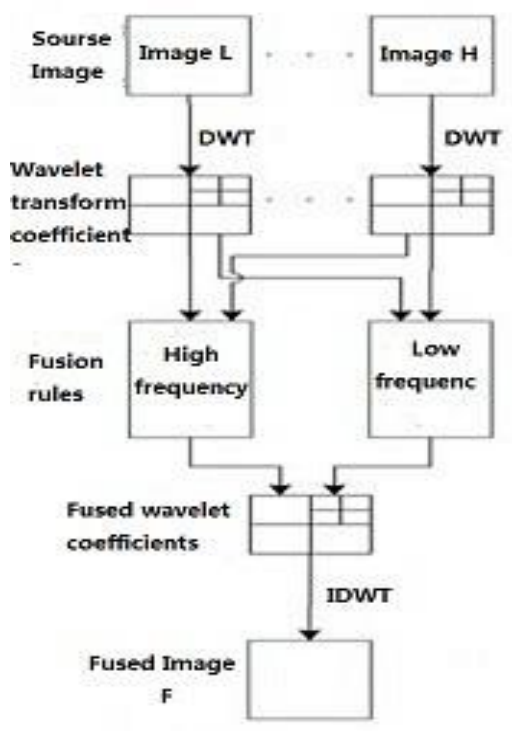

Figure 2. Processes of Image Fusion Algorithm

\section{The Comparison of Experimental Results}

The Laminar flow and core flow are chose as examples, the experimental results of validate fusion algorithm are shown in Table 1.

Table 1. Fusion Comparison

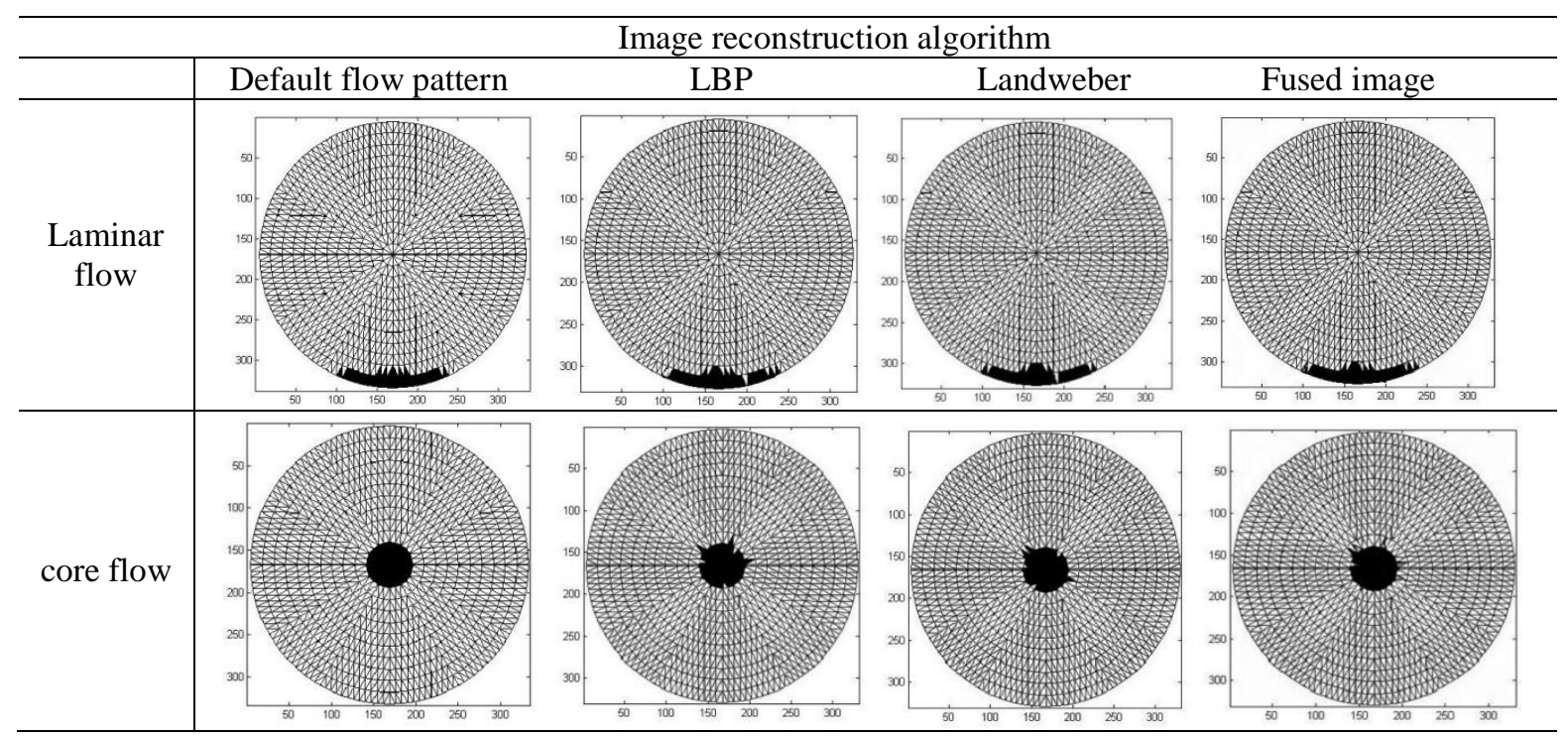

For the results of ECT image fusion, with standard reference image, we can use the standard deviation at first to evaluate the fusion results. The standard deviation is defined as follows:

$$
R M S E=\sqrt{\frac{\sum_{i=1}^{M} \sum_{j=1}^{N}\left[I_{i}(i, j)-I_{F}(i, j)\right]^{2}}{M \times N}}
$$


In this formula, $I_{i}(i, j)$ is the gradation value of the preset image coordinates of point $(i, j), I_{F}(i, j)$ is the gray value corresponding to the coordinates of point $(i, j)$ of the reconstructed image and fusion image, the image size is $M \times N, R M S E$ much smaller, the better fusion effect.

Combination of ECT imaging characteristics, in order to more fully describe the fused image details, we need to adopt the edge retention of the evaluation method to evaluate the fusion results, defined as follows:

$$
E P=\frac{\sum_{m=1}^{M} \sum_{n=1}^{N} Q_{F F}(m, n) \cdot w_{F}(m, n)+Q_{f F}(m, n) \cdot w_{f}(m, n)}{\sum_{m=1}^{M} \sum_{n=1}^{N}\left\{w_{F}(m, n)+w_{f}(m, n)\right\}}
$$

In this formula, $Q_{F F}(m, n)$ and $Q_{f F}(m, n)$ respectively are the edge amplitude and phase angle retention for the default image, the reconstructed image and the fused image, $w_{F}$ and $w_{f}$ are the absolute gradient magnitude for the image $F$ and $f$. The edge amplitude values greater, indicating a better quality of the fused image. The evaluation results are shown in Table 2.

Table 2. Difference of RMSE and EP

\begin{tabular}{c|c|c|c|c}
\hline \multirow{2}{*}{ Algorithm } & \multicolumn{3}{|c}{ Evaluation Index } \\
\cline { 2 - 5 } & \multicolumn{2}{|c}{ RMSE } & \multicolumn{2}{c}{ EP } \\
\cline { 2 - 5 } & Laminar flow & core flow & Laminar flow & core flow \\
\hline LBP & 0.31 & 0.29 & 0.4138 & 0.4297 \\
\hline Landweber & 0.25 & 0.26 & 0.4356 & 0.4389 \\
\hline Fused & 0.22 & 0.24 & 0.4395 & 0.4426 \\
\hline
\end{tabular}

From the table we can see that for ECT, stratified flow and core flow of the ECT typical flow pattern, whether it is from the standard deviation or edge retention, and imaging results of the fusion algorithm is better than LBP algorithm and Landweber algorithm. Especially for the layered stream, the fusion result improved significantly, while the details of the core flow and the energy of the core flow is more concentrated, the effects of several algorithms for the reconstruction of the core flow are better than the effects of the stratified flow.

\section{Conclusion}

In this paper, ECT is combined with image fusion technology, for the ECT imaging characteristics and the characteristics that in the wavelet-based image fusion technology the data can be dealt with separately on a different frequency domain, in order to maximize the enhanced the effect of the reconstructed image, richer and accurate information can be extracted from different source images than a single source image. The effects of the final fusion image can be better improved, if several typical flow of ECT through the objective evaluation of the method, find the best image reconstruction algorithm, and then use the source image which is obtained from different sensors with optimal image reconstruction algorithm to fuse the images.

\section{Acknowledgements}

This work is supported by the National Natural Science Foundation of China(60572153,60972127),Specialized Research Fund for the Doctoral Program of Higher 
Education (200802140001), The Chunhui plan of the Education Ministry (Z2007-1-15013), Natural Science Foundation of Heilongjiang Province(F200609, QC2012C059), Project of Higher Educational Key Laboratory for Measuring and Control Technology and Instrumentations of Heilongjiang Province, Scientific Research Fund of Heilongjiang Provincial Education Department (11541040, 12511097,12531094).

\section{References}

[1] W. G. Pan, Y. Li and X. M. Cao, "Status and Prospects of Process Tomography", Chinese Journal of Scientific Instrument, vol. 25, no. 4, (2004), pp. 1034-1036.

[2] W. Q. Yang and L. Peng, "Image reconstruction algorithms for electrical capacitance tomography", Measurement Science and Technology, vol. 14, no. 1, (2003), pp. 957-1088.

[3] W. Yang, "Design of electrical capacitance tomography sensors", Measurement Science and Technology, vol. 21, no. 4, (2010), pp. 1890-1917.

[4] D. Y. Chen, G. B. Zheng and X. Y. Yu, "Image Reconstruction Algorithm Based on Genetic Algorithms for Two-phase flow Electrical Capacitance Tomography System”, Electric Machines and Control, vol. 9, no. 7, (2003), pp. 207-211.

[5] J. Nunez, Palà and Vicenç, "Multiresolution-based image fusion with additive wavelet decomposition", Geoscience and Remote Sensing, vol. 37, no. 3, (1999), pp. 1204-1211.

[6] G. Pajares and J. Manuel de la Cruz, "A wavelet-based image fusion tutorial A", Pattern Recognition, vol. 37, no. 9, (2004), pp. 1855-1872.

[7] L. Jing, W. Ke and L. Guangxin, "Edge-based image fusion algorithm with wavelet transform", Journal on Communications, vol. 28, no. 4, (2007), pp. 19-23.

[8] P. C. Hansen, "Rank-Deficient and Discrete Ill-Posed Problems", Philadelphia, SIAM Press, (1988).

[9] Yang W. Q. and L. H. Peng, "Image reconstruction algorithms for electrical capacitance tomography", Meas Sci Technol, vol. 14, (2003).

[10] C. Deyun, Y. Congjing and Z. Guibin, "A Novel Method of Plotting Sensor Sensitivity Field and Image Reconstruction Algorithm for Electrical Capacitance Tomography System”, Chinese Journal of Scientific Instrument, vol. 26, no. 3, (2005), pp. 229-234.

[11] C. De-Yun, C. Yu and W. Li-Li, "A novel Gauss-Newton image reconstruction algorithm for Electrical Capacitance Tomography System”, Acta Electronica Sinica, vol. 37, no. 4, (2009), pp. 739-743.

[12] S. Liu, L. Fu and W. Q. Yang, "Prior-online iteration for image reconstruction with electrical capacitance tomography”, IEE Proc-sci.Meas.Technol., vol. 151, no. 3, (2004), pp. 195-200. 